\title{
Prandtl and Rayleigh number dependence of the Reynolds number in turbulent thermal convection
}

\author{
Siegfried Grossmann ${ }^{1, *}$ and Detlef Lohse ${ }^{2, \dagger}$ \\ ${ }^{1}$ Department of Physics, University of Marburg, Renthof 6, D-35032 Marburg, Germany \\ ${ }^{2}$ Department of Applied Physics, University of Twente, 7500 AE Enschede, Netherlands
}

(Received 5 March 2002; published 22 July 2002)

\begin{abstract}
The Prandtl and Rayleigh number dependences of the Reynolds number in turbulent thermal convection following from the unifying theory by Grossmann and Lohse [J. Fluid Mech. 407, 27 (2000); Phys. Rev. Lett. 86, 3316 (2001)] are presented and compared with various recent experimental findings. This dependence $\operatorname{Re}(\operatorname{Ra}, \operatorname{Pr})$ is more complicated than a simple global power law. For $\operatorname{Pr}=5.5$ and $10^{8}<\operatorname{Ra}<10^{10}$ the effective or local power law exponent of $\mathrm{Re}$ as a function of $\mathrm{Ra}$ is definitely less than 0.50 , namely, $\operatorname{Re} \sim \mathrm{Ra}^{0.45}$, in agreement with Qiu and Tong's experimental findings [Phys. Rev. E 64, 036304 (2001)]. We also calculated the kinetic boundary layer width. Both in magnitude and in Ra scaling it is consistent with the data.
\end{abstract}

DOI: 10.1103/PhysRevE.66.016305

PACS number(s): 44.25.+f, 47.27.Te

\section{INTRODUCTION}

Recently, Qiu and Tong [1] measured the Reynolds number $\operatorname{Re} \equiv U L / \nu$ of the large scale velocity ("wind velocity") $U$ in thermal convection as a function of the Rayleigh number $\mathrm{Ra} \equiv \alpha_{p} g L^{3} \Delta / \nu \kappa$ for a fixed Prandtl number $\operatorname{Pr} \equiv \nu / \kappa$ $=5.5$ (water). Here $\alpha_{p}$ is the isobaric thermal expansion coefficient, $g$ the gravitational acceleration, $\Delta$ the temperature difference between the warmer bottom plate and the colder top plate, $L$ the height of the aspect ratio $\Gamma=1$ cell, $\nu$ the kinematic viscosity, and $\kappa$ the thermal diffusivity.

Depending on the precise definition of the wind velocity $U$ one has to consider slightly different Reynolds numbers. Qiu and Tong [1] take a Reynolds number $\mathbf{R e}_{\gamma}$, which is based on a direct measurement of the shear $\gamma$ throughout the cell. Other Reynolds numbers have been based on the frequency peak in the temperature power spectrum $[2,3]$ which has been associated with the large scale convection roll. In the Ra range $10^{8}-10^{10}$ Qiu and Tong $[1,4]$ find the approximate power law $\operatorname{Re}_{\gamma}=0.085 \mathrm{Ra}^{0.455}$ [5]. This exponent is surprising since most hitherto measured data for this Prandtl number or for $\mathrm{Pr}=0.7$ (helium gas) give a power law exponent closer to $0.50[2,3,6]$.

Qiu and Tong [1] also measured the oscillation frequency $f_{0}^{\theta}$ in the time dependence of the temperature crosscorrelation function between the temperatures measured at two points on opposite locations in the Rayleigh-Bénard (RB) cell. These oscillations are considered as a signature of plume transport with the circulating wind, i.e., as a measure of the recurrence rate $U / 2 L$ and thus of the wind velocity $U$ [1]. With this frequency they define a Reynolds number $\operatorname{Re}_{f} \equiv 2 f_{0}^{\theta} L^{2} / \nu$. With $f_{0}^{\theta} \approx U / 2 L$ it can be connected with the standard definition of the Reynolds number $\operatorname{Re}_{f} \approx \approx U L / \nu$ $=\operatorname{Re}$. This Reynolds number $\operatorname{Re}_{f} \theta$ turns out to agree with the above Reynolds number based on the shear rate $\operatorname{Re}_{\gamma}$ $\equiv \gamma L^{2} /(2 \nu)$ (see Fig. 2 of Ref. [1]). Indeed, with $\gamma \approx 2 U / L$ one gets $\operatorname{Re}_{\gamma} \approx \operatorname{Re}$.

\footnotetext{
*Email address: grossmann@physik.uni-marburg.de

${ }^{\dagger}$ Email address: lohse@tn.utwente.nl
}

In a very recent report Lam et al. [7] also present experimental results for the Rayleigh number dependence of the Reynolds number, but in addition also for its Prandtl number dependence. For $10^{8}<\mathrm{Ra}<3 \times 10^{10}$ they find the effective scaling law $\operatorname{Re} \sim \operatorname{Ra}^{0.43} \operatorname{Pr}^{-0.76}$ for a Reynolds number based on the frequency $f_{0}^{u}$ extracted from the autocorrelation function of the velocity fluctuations, $4 f_{0}^{u} L^{2} / \nu$. Note that this expression differs by a factor of 2 from that of Ref. [1]. To allow for easier comparison, we drop this factor 2 and define $\operatorname{Re}_{f^{u}} \equiv 2 f_{0}^{u} L^{2} / \nu$ which, using the data from [7], is $\operatorname{Re}_{f u}$ $=0.545 \mathrm{Ra}^{0.43} \mathrm{Pr}^{-0.76}$. The $R a$ dependence is consistent with Qiu and Tong's findings $\operatorname{Re}_{\gamma}=\operatorname{Re}_{f}=0.085 \operatorname{Ra}^{0.455}[1,4]$. Also the absolute values agree rather well: For water $(\operatorname{Pr}=5.5)$ at $\mathrm{Ra}=10^{9}$ one has $\mathrm{Re}_{f}=1106$ according to [7] and $\operatorname{Re}_{f} \theta$ $=1058$ according to [1].

In addition, Lam et al. [7] measure a Reynolds number based on the velocity maximum in the cell, $\operatorname{Re}_{U, \max }$ $\equiv U_{\max } L / \nu$. The $R a$ exponent of that Reynolds number appears to vary as a function of the Prandtl number from 0.50 to 0.68 as Pr increases from 3 to 1200 . The power law exponent is definitely steeper than that for the velocity autocorrelation based Reynolds number $\operatorname{Re}_{f u}$.

\section{THE REYNOLDS NUMBER}

These experimental findings have triggered us to finally present the Ra and Pr number dependence of Re, as it follows from our unifying theory of thermal convection $[8,9]$.

The main idea of that theory is to decompose the energy dissipation rate $\epsilon_{u}$ and the thermal dissipation rate $\epsilon_{\theta}$ into their boundary layer (BL) and bulk contributions,

$$
\begin{gathered}
\epsilon_{u}=\epsilon_{u, B L}+\epsilon_{u, \text { bulk }}, \\
\epsilon_{\theta}=\epsilon_{\theta, B L}+\epsilon_{\theta, \text { bulk }} .
\end{gathered}
$$

For the left-hand sides the exact relations $\epsilon_{u}=\left(\nu^{3} / L^{4}\right)(\mathrm{Nu}$ -1) $\operatorname{RaPr}^{-2}$ and $\epsilon_{\theta}=\kappa\left(\Delta^{2} / L^{2}\right), \mathrm{Nu}$ are employed, where $\kappa$ is the thermal diffusivity and $\Delta$ the temperature difference between the bottom and top plates. The individual contributions on the right-hand sides of Eqs. (1) and (2) are modeled 
in terms of the large scale velocity $U$, the temperature difference $\Delta$, the height $L$, and the widths $\lambda_{u}$ and $\lambda_{\theta}$ of the kinetic and thermal boundary layers (see Refs. [8,9]). Note that $\epsilon_{u, B L}$ contains contributions from both the top and bottom plates as well as from the sidewalls. From visualizations of the thermal boundary layers we conclude that $\epsilon_{\theta, B L}$ mainly contains top and bottom plate contributions as the sidewalls are thermally isolated. The thermal dissipation in the sidewall boundary layers scales like the bulk dissipation, which is proportional to $\mathrm{RePr}$, and therefore can be included into $\epsilon_{\theta, \text { bulk }}[10]$. For the thicknesses of the thermal and kinetic boundary layers we assume

$$
\lambda_{\theta}=L /(2 \mathrm{Nu})
$$

and

$$
\lambda_{u}=a L / \sqrt{\operatorname{Re}},
$$

respectively. The latter expression holds in laminar boundary layers of Prandtl-Blasius type [see [11] or [12], Sec. 39, Eq. (39.12)]. In Refs. [8,9] we adopted $a=1 / 4$ for the dimensionless prefactor that determines the absolute width of the kinetic boundary layer. The final result is [see Eqs. (13) and (14) of Ref. [9]]:

$$
\begin{gathered}
\mathrm{Nu} \operatorname{RaPr}^{-2}=c_{1} \frac{\mathrm{Re}^{2}}{g\left(\sqrt{\operatorname{Re}_{c} / \operatorname{Re}}\right)}+c_{2} \operatorname{Re}^{3}, \\
\mathrm{Nu}=c_{3} \operatorname{Re}^{1 / 2} \operatorname{Pr}^{1 / 2}\left[f\left(\frac{2 a \mathrm{Nu}}{\sqrt{\operatorname{Re}_{c}}} g\left(\sqrt{\frac{\operatorname{Re}_{c}}{\operatorname{Re}}}\right)\right)\right]^{1 / 2} \\
+c_{4} \operatorname{Pr} \operatorname{Re} f\left(\frac{2 a \mathrm{Nu}}{\sqrt{\operatorname{Re}_{c}}} g\left(\sqrt{\frac{\operatorname{Re}_{c}}{\operatorname{Re}}}\right)\right) .
\end{gathered}
$$

The crossover functions $f$ and $g$ model the crossover from the thermal boundary layer nested in the kinetic one towards the inverse situation and from Eq. (4) toward $\lambda_{u} \sim L$, respectively; for details, see Ref. [9]. The four prefactors $c_{i}, i$ $=1,2,3,4$, and the transition Reynolds number $\operatorname{Re}_{c}$ to the large Pr regime (cf. [9]) were fitted to 151 experimental data points $\mathrm{Nu}(\mathrm{Ra}, \mathrm{Pr})$, measured by Ahlers and $\mathrm{Xu}$ [13] for an aspect ratio $\Gamma=1$ cell, still assuming $a=0.25$.

Note that once $a$ is chosen and the $c_{i}$ and $\operatorname{Re}_{c}$ are fixed through the fit, not only is $\mathrm{Nu}(\mathrm{Ra}, \mathrm{Pr})$ determined for all $\mathrm{Ra}$ and $\mathrm{Pr}$, but also $\mathrm{Re}(\mathrm{Ra}, \mathrm{Pr})$. However, while $\mathrm{Nu}(\mathrm{Ra}, \mathrm{Pr})$ does not depend on the choice of $a=1 / 4$, the wind amplitude $\operatorname{Re}(\mathrm{Ra}, \operatorname{Pr})$ does depend on the value of $a$. The reason is the invariance of Eqs. (5) and (6) under the following rescaling transformation: $\operatorname{Re} \rightarrow \alpha \operatorname{Re}, \quad \operatorname{Re}_{c} \rightarrow \alpha \operatorname{Re}_{c}, \quad a \rightarrow \alpha^{1 / 2} a, \quad c_{1}$ $\rightarrow c_{1} / \alpha^{2}, c_{2} \rightarrow c_{2} / \alpha^{3}, c_{3} \rightarrow c_{3} / \alpha^{1 / 2}$, and $c_{4} \rightarrow c_{4} / \alpha$.

As meanwhile reliable results for $\operatorname{Re}(\mathrm{Ra}, \mathrm{Pr})$ are known, we use this amplitude rescaling freedom in Eqs. (5) and (6) to fix the prefactor $a$ and thus the width of the kinetic boundary layer. This procedure does not affect the functional dependence of Re on Ra and Pr. It only fixes an overall factor for $\operatorname{Re}(\operatorname{Ra}, \operatorname{Pr})$. The Nusselt number, in contrast, is not affected at all.

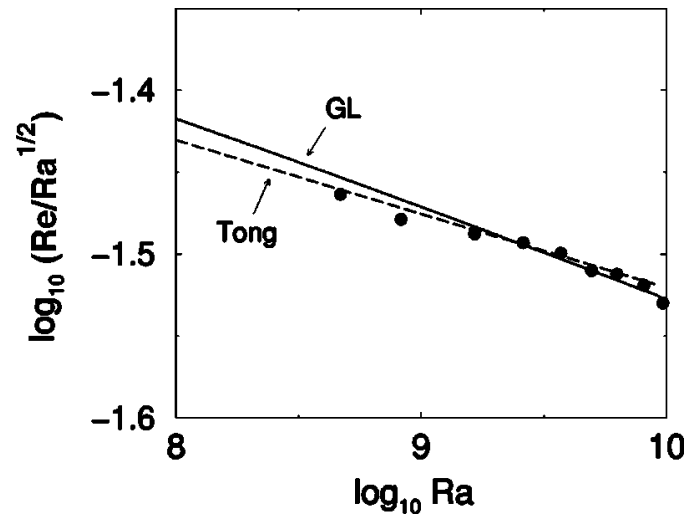

FIG. 1. $\mathrm{Re} / \mathrm{Ra}^{1 / 2}$ vs $\mathrm{Ra}$ for $\operatorname{Pr}=5.5$ from the experiments of Ref. [1] (thick dots and dashed line as best fit $\mathrm{Re}=0.085 \mathrm{Ra}^{0.455}$ ) and from the theory of Ref. [9] with $a=0.482$ (solid line). A local power law fit to the theoretical result in the Ra regime shown gives $\mathrm{Re}=0.102 \mathrm{Ra}^{0.447}$.

To determine the prefactor $a$, we take Qiu and Tong's experimental finding $\mathrm{Re}=R e_{\gamma}=0.085 \mathrm{Ra}^{0.455}$ [1]; see Fig. 1. The result is $a=0.482$ rather than $a=0.25$ as was suggested in [12] and also assumed in our earlier publications $[8,9]$. With this choice of $a$, the coefficients in Eqs. (5) and (6) are $c_{1}=8.7, c_{2}=1.45, c_{3}=0.46, c_{4}=0.013$, and $\operatorname{Re}_{c}$ $=1.0{ }^{1}$

In Fig. 1 we show $\mathrm{Re} / \mathrm{Ra}^{1 / 2}$ vs $\mathrm{Ra}$ as resulting from the theory together with the measured data of Refs. $[1,4]$. Indeed, the theoretical data for $\operatorname{Re}(\mathrm{Ra})$ also display a local power law exponent which is significantly smaller than $1 / 2$. A power law fit in the $\mathrm{Ra}$ regime $10^{8}-10^{10}$ gives $\mathrm{Re}$ $=0.102 \mathrm{Ra}^{0.447}$, with a power law exponent very close to what has been observed by Qiu and Tong [1].

For completeness we also show again the phase diagram in the Ra-Pr space (see Fig. 2). This is identical to Fig. 1 of Ref. [9], with two exceptions. (i) Some additional experimental data points have been added. (ii) As we now took $a$ $=0.482$ rather than $a=1 / 4$ in relation (4) in order to adapt the overall factor in Re to the measured values of Fig. 1, the shear Reynolds number of the kinetic boundary layer

$$
\operatorname{Re}_{s}=\lambda_{u} U / \nu=a \sqrt{\operatorname{Re}}
$$

also changes as compared to [9]. For a given wind Reynolds number $\operatorname{Re}$ the shear Reynolds number $\operatorname{Re}_{s}$ increases with $a$. Now, since according to [12], Sec. 41, the kinetic BL suffers a transition from laminar to turbulent for $\mathrm{Re}_{s} \approx 420$, the larger choice of $a$ implies that the onset of shear triggered turbulence in the BL happens already for smaller Re. Therefore the dotted line in the phase diagram, which signals this onset of turbulence in the kinetic BL, shifts upward. ${ }^{2}$ The

\footnotetext{
${ }^{1}$ Applying the above rescaling of $\operatorname{Re}, \operatorname{Re}_{c}$, and the $c_{i}$, these numbers exactly correspond to the numbers given in [9]. The rescaling factor to be taken is $\alpha=(a / 0.25)^{2}=3.72$.

${ }^{2}$ Note again that all heat current plots $\mathrm{Nu}(\mathrm{Ra}, \mathrm{Pr})$ of Ref. [9] remain completely unchanged through the modification of the value $a$ in Eq. (4).
} 


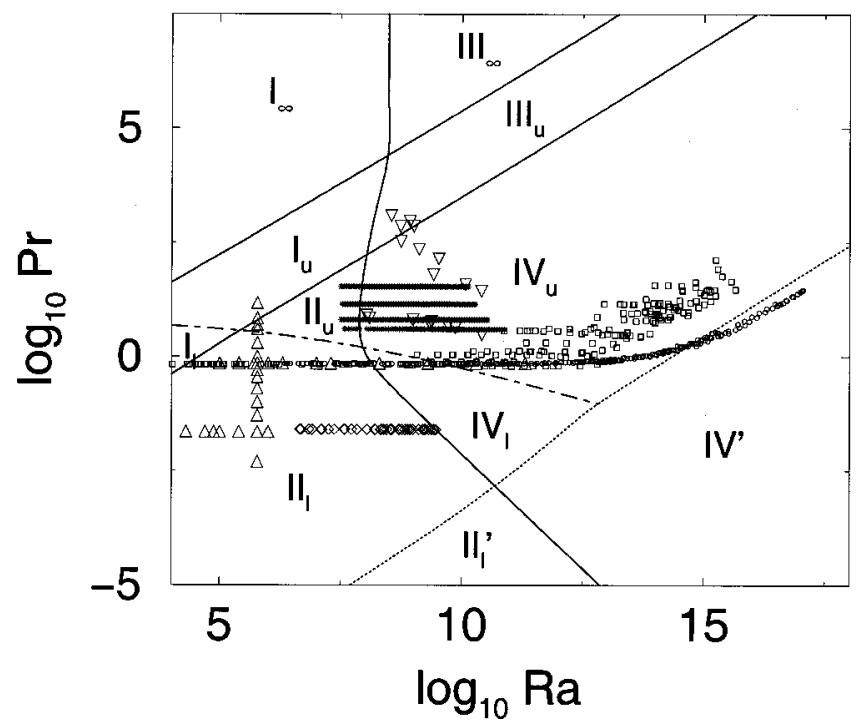

FIG. 2. Phase diagram in the Ra-Pr plane according to the theory of Ref. [9] The upper solid line means $\operatorname{Re}=\operatorname{Re}_{c}$, the lower nearly parallel solid line is $\epsilon_{u, B L}=\epsilon_{u, b u l k}$, the curved solid line is $\epsilon_{\theta, B L}=\epsilon_{\theta, \text { bulk }}$, and the long-dashed line is the line $\lambda_{u}=\lambda_{\theta}$. The dotted line indicates where the laminar kinetic BL becomes turbulent, based on a critical shear Reynolds number $\operatorname{Re}_{s}^{*} \approx 420$ of the kinetic BL. Beyond that line, the definition (4) of $\lambda_{u}$ does not make sense anymore, and therefore no line $\lambda_{u}=\lambda_{\theta}$ is drawn there. Data points where $\mathrm{Nu}$ has been measured or numerically calculated have been included (for several aspect ratios): squares Chavanne et al. [14]; diamonds; Cioni et al. [15]; circles Niemela et al. [16]; stars; Ahlers and $\mathrm{Xu}$ [13]; down triangles, Xia et al. [17]; up triangles, Verzicco and Camussi (numerical simulations) [18].

location of this (dotted) turbulence transition line is discussed at the end of this paper in more detail.

The phase diagram Fig. 2 offers an understanding of why the scaling exponent of $\mathrm{Re}$ with $\mathrm{Ra}$ is smaller than 0.50 for $\operatorname{Pr}=5.5$. The exponent 0.447 originates in the combined contributions from the regimes $\mathrm{IV}_{u}$ (with the pure power law exponent 4/9), $\mathrm{IV}_{l}$ (with the pure power law exponent $1 / 2$ ), and $\mathrm{II}_{u}$ and $\mathrm{II}_{l}$ (with the pure power law exponents $2 / 5$ ). Incidentally, the (unweighted) mean of the scaling exponents $4 / 9,1 / 2,2 / 5$, and $2 / 5$ in the Ra range $10^{8}-10^{10}$ gives 0.44 , very close to the numerical finding in Fig. 1. For larger Ra, beyond $10^{10}$, the influence of the regimes $\mathrm{II}_{l, u}$ will decrease and that of $\mathrm{IV}_{u, l}^{\prime}$ will become stronger. Thus we expect a larger local exponent. If $\mathrm{IV}_{l}$ (with $1 / 2$ ), $\mathrm{IV}_{u}$ (with 4/9), and $\mathrm{IV}_{u}^{\prime}$ (with 1/2) dominate, the unweighted mean is 0.48 . We emphasize that generally there is no pure power law behavior but, instead, a more general dependence of $\mathrm{Re}$ on $\mathrm{Ra}$, cf. Fig. 3.

For other Pr our theory predicts a local exponent $1 / 2$. In Fig. 3(a) we have plotted $\mathrm{Re} / \mathrm{Ra}^{1 / 2}$ vs $\mathrm{Ra}$ for various $\mathrm{Pr}$. The local exponent $1 / 2$ can be noticed, e.g., for small $\mathrm{Pr}$ $\approx 10^{-3}-10^{-4}$ and large $\mathrm{Ra} \approx 10^{13}\left(\right.$ regime $\left.\mathrm{IV}_{u}\right)$, or for large $\mathrm{Pr} \approx 10^{2}$ and small $\mathrm{Ra} \approx 10^{6}$ (regime $\mathrm{I}_{u}$ ).

It is evident from Fig. 3(a) that there is no pure power law throughout the whole Ra regime. As stressed in Refs. [8,9], power laws only apply locally. The same holds for the dependence of Re on Pr. In Fig. 3(b) we show this dependence
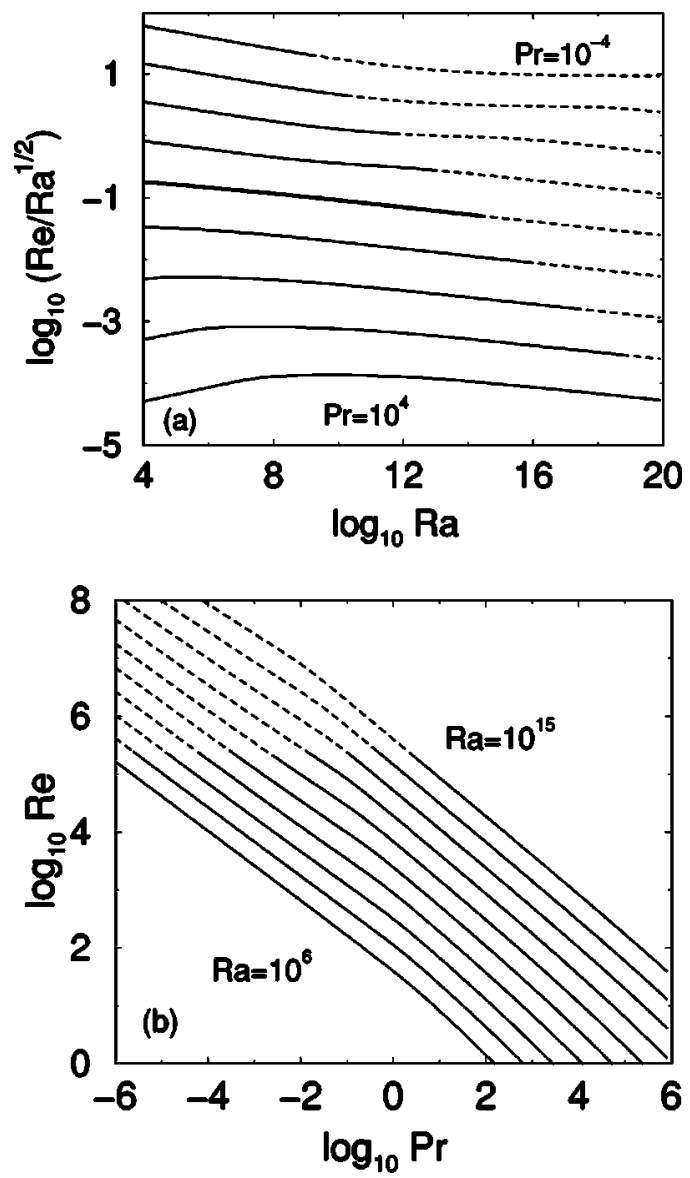

FIG. 3. (a) $\mathrm{Re} / \mathrm{Ra}^{1 / 2}$ vs Ra for various $\mathrm{Pr}$ and (b) Re vs Pr for various $\mathrm{Ra}$, according to our unifying theory. The dashed parts of the lines indicate the region beyond the expected transition of the kinetic BL into the turbulent state. There the $\mathrm{II}_{l}^{\prime}, \mathrm{IV}_{l}^{\prime}$, and $\mathrm{IV}_{u}^{\prime}$ exponents will influence and change the curves: $N u \propto \operatorname{Ra}^{1 / 2} \operatorname{Pr}^{1 / 2}$ and $\operatorname{Re} \propto \operatorname{Ra}^{1 / 2} \operatorname{Pr}^{-1 / 2}$, as in $\mathrm{IV}_{l}$.

as it results from our theory. The Reynolds number strongly decreases with increasing Pr. This reflects the increasing effects of the viscosity on the wind as Pr grows.

The absence of global scaling exponents is highlighted in Fig. 4 in which we present the local slopes $d \log _{10}(\mathrm{Re}) / d \log _{10}(\mathrm{Ra})$ and $d \log _{10}(\mathrm{Nu}) / d \log _{10}(\mathrm{Ra})$ resulting from our theory: Indeed, neither one of these local scaling exponents is constant over a larger range for hitherto experimentally achievable Rayleigh and Prandtl numbers.

We now compare our results for $\operatorname{Re}(\mathrm{Ra}, \mathrm{Pr})$ with the recent experimental findings by Lam et al. [7] in the regime $10^{8}$ $<\operatorname{Ra}<3 \times 10^{10}$ and $3<\operatorname{Pr}<1200$. For this Prandtl number regime we find [cf. Fig. 3(b)] effective (local) power laws $\mathrm{Re} \sim \mathrm{Pr}^{-0.70}$ for $\mathrm{Ra}=10^{10}$ and $\mathrm{Re} \sim \mathrm{Pr}^{-0.73}$ for $\mathrm{Ra}=10^{8}{ }^{3}$. The corresponding experimental local power law exponent of the Reynolds number $\operatorname{Re}_{f u}$ based on the oscillation frequency $f_{0}^{u}$

\footnotetext{
${ }^{3}$ These exponents can be understood as originating from the physics in regime $\mathrm{IV}_{u}$ (with the Prandtl dependence exponent -2/3) together with some influence of the regimes $\mathrm{I}_{u}$ and $\mathrm{II}_{u}$ (with $\mathrm{Pr}$ exponents $-5 / 6$ and $-2 / 3$ ).
} 

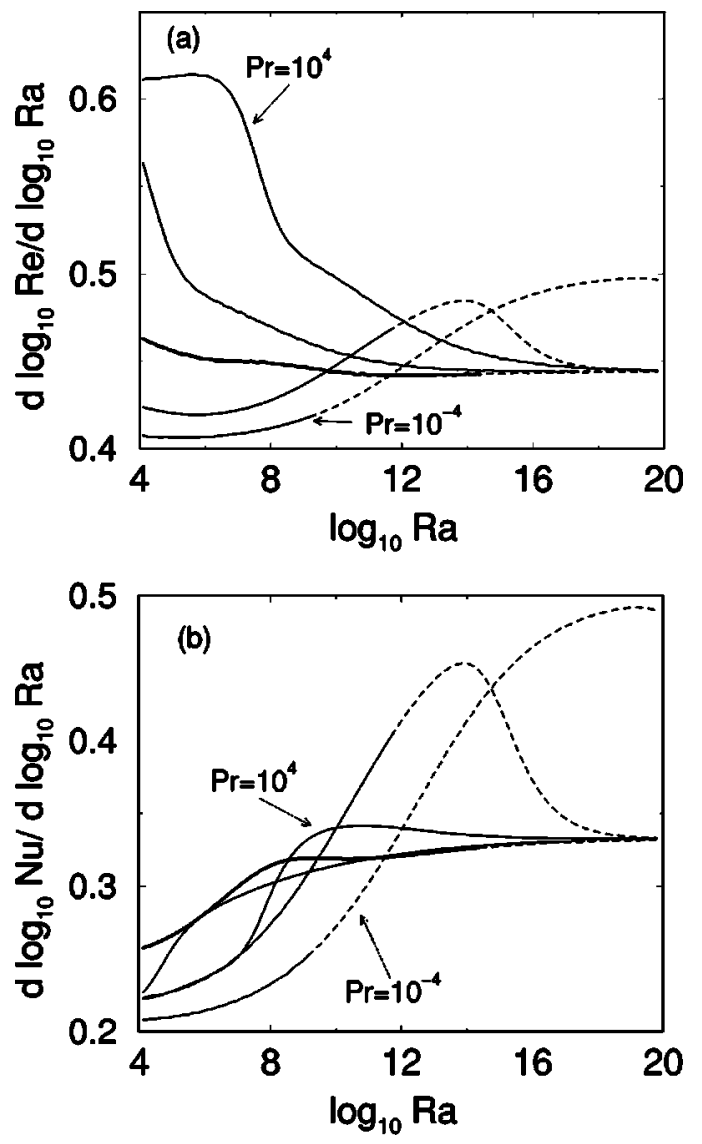

FIG. 4. Local slopes $d \log _{10}(\mathrm{Re}) / d \log _{10}(\mathrm{Ra}) \quad$ (a) and $d \log _{10}(\mathrm{Nu}) / d \log _{10}(\mathrm{Ra})$ (b) for five Prandtl numbers $\operatorname{Pr}=10^{-4}$, $\operatorname{Pr}=10^{-2}, \operatorname{Pr}=10^{\circ}$ (thick line), $\operatorname{Pr}=10^{2}$, and $\operatorname{Pr}=10^{4}$ as they result from our theory. As in Fig. 3 the dashed parts of the lines indicate the region beyond the expected transition of the kinetic BL into the turbulent state.

is $-0.76 \pm 0.01$. For the maximum velocity based Reynolds number $\operatorname{Re}_{U, \max }$ the corresponding exponent varies between -0.88 and -0.94 . Vice versa, for the Rayleigh number regime $10^{8}<\mathrm{Ra}<3 \times 10^{10}$ we find [see Fig. 3(a)] effective (local) power laws $\mathrm{Re} \sim \mathrm{Ra}^{0.44}$ for $\mathrm{Pr}=3$ and $\mathrm{Re} \sim \mathrm{Ra}^{0.48}$ for $\mathrm{Pr}$ $=1200$. Lam et al.'s [7] experimental local exponents are 0.43 for an oscillation based Reynolds number $\mathrm{Re}_{f^{u}}$ (consistent with the findings from Qiu and Tong for $\operatorname{Re}_{f} \theta$ and between 0.50 and 0.68 for the maximum velocity based Reynolds number $\operatorname{Re}_{U, \max }$.

To summarize, our theory is in good agreement with (i) the Reynolds number $\operatorname{Re}_{f u}$ based on the circulation frequency $f_{0}^{u}$ as measured in [7], (ii) the Reynolds number $\operatorname{Re}_{f} \theta$ based on the circulation frequncy $f_{0}^{\theta}$ as measured in $[1,4]$, and (iii) the Reynolds number $\operatorname{Re}_{\gamma}$ based on the shear rate $\gamma_{h}$ as measured in [1] [which agrees with the method (ii) according to [1]]. In contrast, the Reynolds number $\operatorname{Re}_{U, \max }$ based on the maximum velocity as measured in [7] shows a slightly stronger dependence on both $\mathrm{Ra}$ and $\mathrm{Pr}$ than is suggested by our theory.

Our theory (as yet) does not distinguish between these various Reynolds numbers. For $\mathrm{Ra} \rightarrow \infty$ one would expect that they all show the same scaling behavior, but at finite and
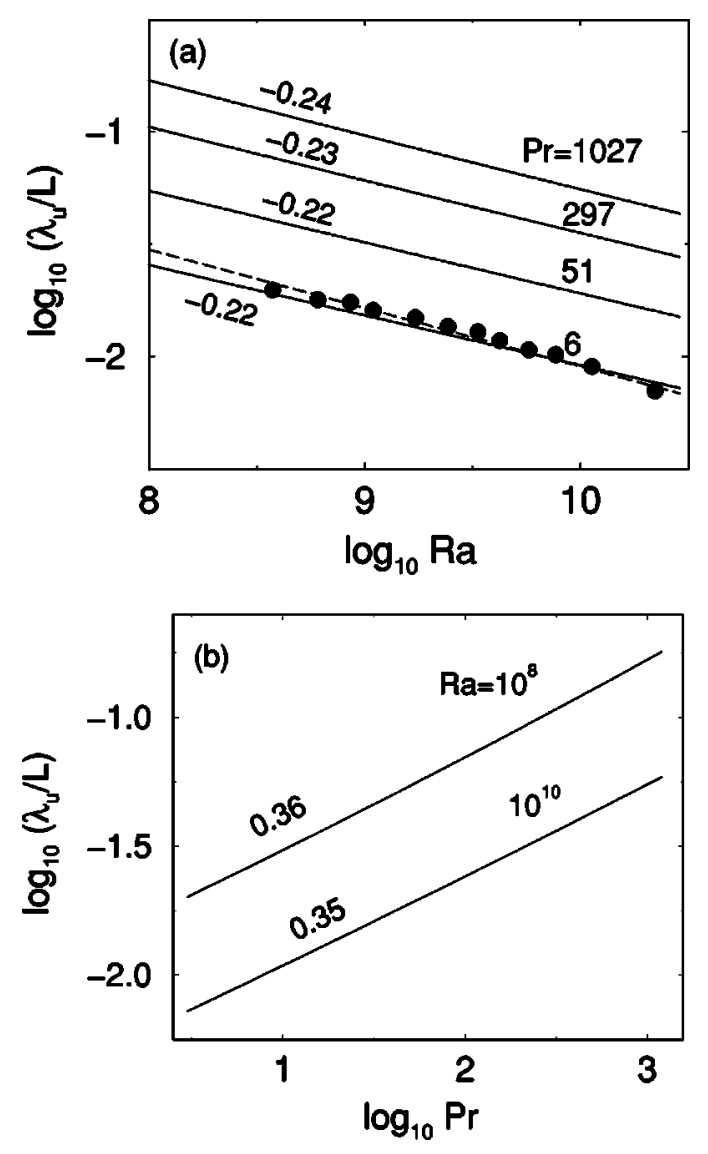

FIG. 5. $\lambda_{u}(\mathrm{Ra}, \operatorname{Pr}) / L$, according to our unifying theory, in the same regime $10^{8}<\operatorname{Ra}<3 \times 10^{10}$ (a) and $3<\operatorname{Pr}<1200$ (b) as experimentally examined by Lam et al. [7]. The numbers on the curves are the local power law exponents of the solid curves. The dashed line is the experimental finding $\lambda_{u} / L=3.6 \mathrm{Ra}^{-0.26}[19]$ for the sidewall thickness of the kinetic boundar layer for $\operatorname{Pr} \approx 7$. The data points measured in that publication [19] are given as filled circles.

relatively small $\mathrm{Ra} \approx 10^{8}-10^{10}$ deviations might occur, which apparently is the case here.

\section{KINETIC BOUNDARY LAYER THICKNESS}

Next, we compare with the measured thickness of the top and bottom kinetic boundary layers in an aspect ratio $\Gamma=1$ cell [7]. For the regime $10^{8}<\mathrm{Ra}<3 \times 10^{10}$ and $3<\operatorname{Pr}$ $<1200$, the experimental results can be parametrized in the local power law $\lambda_{u} / L=0.65 \mathrm{Ra}^{-0.16 \pm 0.02} \operatorname{Pr}^{0.24 \pm 0.01}$. Our theory gives steeper dependences on both Ra and $\operatorname{Pr}$ (see Fig. 5). The correspinding theoretical exponents are around -0.23 and 0.35 . For the Ra dependence the discrepancy between the experimental Ra scaling exponent -0.16 and the theoretical Ra scaling exponent -0.23 (for fixed $\operatorname{Pr} \approx 6$ ) was already identified in [8], when compared to older experimental results [20].

However, note that the kinetic boundary layer thickness measured at the sidewalls does agree with the prediction from our theory. For that thickness Qiu and Xia [19] found $\lambda_{u} / L=3.6 \mathrm{Ra}^{-0.26 \pm 0.03}$ for $\mathrm{Pr}=7$, which we also drew in Fig. 5. The agreement with the theoretical prediction is very 
good. Note that the theoretical overall prefactor was adapted to a different quantity (namely, Re; see Fig. 1) in a different experiment (cf. [1]) and therefore the quality of the agreement not only of the slope but also of the absolute size must be emphasized.

As the theoretical Ra scaling exponent for the BL thickness is closer to the experimental result for the sidewall $\mathrm{BL}$ rather than to that of the top or bottom $\mathrm{BL}$, it seems that the main contribution to the kinetic energy dissipation in the boundary layers comes from the sidewalls and not from the top or bottom plates. Given that for an aspect ratio $\Gamma=1$ (cylindrical) cell (i.e., the diameter equals its height $L$ ) the sidewalls contribute twice the top plus bottom area, this may not be too surprising. If we weigh the experimental scaling exponents of side and top/bottom walls according to the relative areas, we obtain $(-0.16-2 \times 0.26) / 3=-0.23$ (for $\operatorname{Pr}$ $=7$ ), very close to our theoretical value. Since our theory does not distinguish between the top/bottom plates and the sidewalls in Eqs. (1) and (4), there is no freedom for different exponents in the theory and an averaged value results. A refinement of the theory, including a possible physical explanation of the different $\mathrm{Ra}$ scaling of the sidewall and the bottom/top kinetic boundary layer widths (as an effect of the geometrical shape of the RB cell) and a first understanding of the aspect ratio dependence (as coming from the observation that the boundary layer probes the streamwise extension of the surface rather than the perpendicular length scale of the cell; cf. [11]) will be published in a forthcoming paper [10].

\section{BREAKDOWN OF THE LAMINAR KINETIC BOUNDARY LAYER}

One expects that at large enough Ra the laminar kinetic BLs will become turbulent due to the shear triggered transition. Since the sidewall and top/bottom BL thicknesses seem to scale differently, this may in fact happen at different Ra. Note also that the different Ra scaling of the sidewall and the top/bottom BL thicknesses implies an aspect ratio dependence of the kinetic laminar BL breakdown.

Our theory does not distinguish between sidewalls and top/bottom plates, and the onset of the shear driven turbulence, indicated by the dotted line in the phase diagram Fig. 2 , is based on only one $\lambda_{u}$ [Eq. (4)]. As onset Reynolds number we took a critical shear Reynolds number of $\mathrm{Re}_{s}^{*}$ $=420$, which is the value advocated in Ref. [12] for a semiinfinite plane boundary layer. For $\operatorname{Pr}=1$ this means the onset of shear turbulence at $\mathrm{Ra}=3 \times 10^{14}$. For the finite and closed $\mathrm{RB}$ geometry other values for $\mathrm{Re}_{s}^{*}$ might be more realistic. If we take the critical shear Reynolds number $\mathrm{Re}_{s}^{*}$ only half as large (i.e., $\mathrm{Re}_{s}^{*}=210$ ), the onset of shear driven turbulence in the boundary layers takes place already at $\mathrm{Ra}=10^{13}$ (again for $\operatorname{Pr}=1)$. Or, for fixed $\mathrm{Ra}$, reducing $\mathrm{Re}_{s}^{*}$ by a factor of $1 / 2$ leads to an upward shift of the dotted line in the Pr direction by a factor of about 10 .

The breakdown of the top and bottom laminar kinetic BLS is expected to lead to an enhanced heat transfer. In the of Chavanne et al. experiments [14,21] such an enhanced heat transfer is indeed observed around $\mathrm{Ra}=10^{11}$. In con-

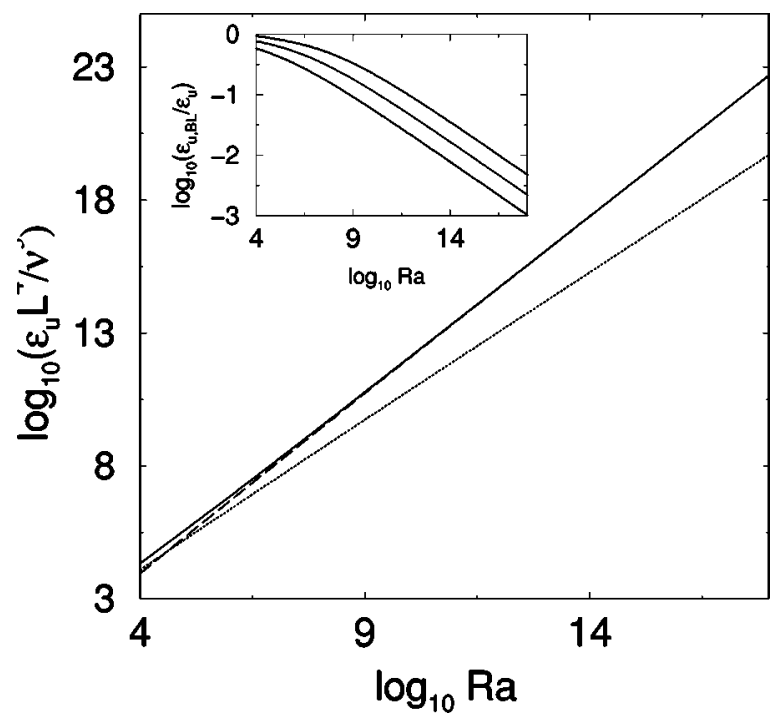

FIG. 6. The kinetic energy $\epsilon_{u}$ (solid), the kinetic energy in the bulk $\epsilon_{u, b u l k}$ (dashed), and the kinetic energy in all kinetic boundary layers $\epsilon_{u, B L}$ (dotted) as a function of $\operatorname{Ra}$ for $\operatorname{Pr}=1$. As in the text, all three functions are made dimensionless with $L$ and $\nu$, e.g., $\epsilon_{u} /\left(\nu^{3} L^{-4}\right)=\operatorname{Pr}^{-2} \operatorname{Ra}(\mathrm{Nu}-1)$. The inset shows the ratio $\epsilon_{u, B L} / \epsilon_{u}$ as a function of $\operatorname{Ra}$ for $\operatorname{Pr}=1,10$, and 100 (bottom to top).

trast, the experiments of Niemala et al. [16] do not display such an enhancement. Our theory cannot resolve this discrepancy between the experiments. On the contrary, the phase diagram Fig. 2 suggests that it is the data of [16] in which a breakdown of the laminar kinetic BL should be visible (if at all), as they reach larger Ra. More work will be necessary to resolve this paradox.

The authors thank J. Niemela, K. Sreenivasan, P. Tong, and K. Q. Xia for very helpful discussions and for making their data and their reports available to us prior to publication. The work is part of the research program of FOM, which is financially supported by NWO. It was also supported by the European Union (EU) under Contract No. HPRN-CT-2000-00162 and by the German-Israeli Foundation (GIF).

\section{APPENDIX: KINETIC DISSIPATION IN THE BULK AND IN THE BL}

In order to give an idea of the absolute kinetic energy dissipation rates per mass and their distribution between bulk and boundary layers, we calculated [from Eq. (5)] Fig. 6, showing $\epsilon_{u}, \epsilon_{u, b u l k}$, and $\epsilon_{u, B L}$ as functions of Ra (here for $\operatorname{Pr}=1)$. With typical values such as $\nu=10^{-6} \mathrm{~m}^{2} \mathrm{~s}^{-1}$ and $L$ $=0.5 \mathrm{~m}$ one gets, for $\mathrm{Ra}=10^{10}$, the small dissipation rate $\epsilon_{u}=1.6 \times 10^{-5} \mathrm{~m}^{2} \mathrm{~s}^{-3}$. For $\mathrm{Ra}=10^{18}$ one a obtains in contrast the large value $\epsilon_{u}=8.0 \times 10^{5} \mathrm{~m}^{2} \mathrm{~s}^{-3}$. This cannot, of course, be realized in a laboratory. Very large Ra in laboratory experiments are reached today by pressure induced increase of the density, which in turn reduces the kinetic and thermal diffusivities $\nu$ and $\kappa$. This leads to reasonably moderate dissipation rates. In the experiment of [16], for instance, for $\mathrm{Ra}=1.06 \times 10^{10}$ it is $\nu=5.04 \times 10^{-6} \mathrm{~m}^{2} \mathrm{~s}^{-1}, \operatorname{Pr}$ $=0.689$, and thus $\epsilon_{u}=7 \times 10^{-3} \mathrm{~m}^{2} \mathrm{~s}^{-3}$, while for $\mathrm{Ra}=1.26$ 
$\times 10^{16}$ it is $\nu=3.245 \times 10^{-8} \mathrm{~m}^{2} \mathrm{~s}^{-1}, \mathrm{Pr}=7.36$, and $\epsilon_{u}=1.5$ $\times 10^{-3} \mathrm{~m}^{2} \mathrm{~s}^{-3}$, still rather moderate. For these relatively small Pr, the boundary layers' contributions to the total kinetic dissipation rates are rather small, as one could anticipate from Fig. 2.

Interestingly enough, if the $\mathrm{Ra}$ increase is obtained by enlargening the density and so reducing $\nu$ and $\kappa$, the dissipation rate becomes smaller with increasing Ra. If all other parameters defining $\mathrm{Ra}$, such as $\alpha_{p}, g, \Delta$, and $L$ are kept fixed, one evaluates $\epsilon_{u} \propto\left[\left(\alpha_{p} g \Delta\right)^{3} L\right]^{1 / 2} \operatorname{Pr}^{-1 / 2} \mathrm{Ra}^{\beta-1 / 2}$. Here $\beta$ denotes the $\mathrm{Nu}$ versus $\mathrm{Ra}$ scaling exponent. Using $\beta$ $\approx 1 / 3$ we find $\epsilon_{u} \propto\left[\left(\alpha_{p} g \Delta\right)^{3} L\right]^{1 / 2} \operatorname{Pr}^{-1 / 2} \mathrm{Ra}^{-1 / 6}$.
[1] X.L. Qiu and P. Tong, Phys. Rev. Lett. 87, 094501 (2001).

[2] B. Castaing, G. Gunaratne, F. Heslot, L. Kadanoff, A. Libchaber, S. Thomae, X. Wu, S. Zaleski, and G. Zanetti, J. Fluid Mech. 204, 1 (1989).

[3] E.D. Siggia, Annu. Rev. Fluid Mech. 26, 137 (1994).

[4] X.L. Qiu and P. Tong, Phys. Rev. E 64, 036304 (2001).

[5] The prefactor and exponent are based on the latest measurements by Tong and co-workers (private communication); the values given in the publications [1] and [4] are slightly different. Note that the prefactor very sensitively depends on the power: At $\mathrm{Ra}=10^{10}$ a difference of 0.005 in the power means a change by a factor of 1.12 in the prefactor.

[6] J. Niemela, L. Skrbek, K.R. Sreenivasan, and R.J. Donnelly, J. Fluid Mech. 449, 169 (2001).

[7] S. Lam, X. D. Shang, S. Q. Zhou, and K. Q. Xia, Phys. Rev. E (to be published).

[8] S. Grossmann and D. Lohse, J. Fluid Mech. 407, 27 (2000).

[9] S. Grossmann and D. Lohse, Phys. Rev. Lett. . 86, 3316 (2001).

[10] S. Grossmann and D. Lohse, (unpublished).

[11] The Navier-Stokes based approximate equations to describe laminar boundary layer flow near a plane surface of streamwise extension $\ell$ were given first in 1904 by Ludwig Prandtl; see L. Prandtl, Verhandlungen des III. Int. Math. Kongr., Heidelberg 1904, (Teubner, Leipzig, 1905), p. 484; see also Ludwig Prandtl, Ges. Abhandlungen, edited by W. Tollmien et al. (Springer, Berlin, 1961), Vol. 2, p. 575. Since we consider aspect ratio $\Gamma=1$, we can use the cell height $L$ instead of the cell width $\ell$. In 1908, in his thesis, Paul Richard Heinrich Blasius extended the laminar boundary layer theory to semiinfinite surfaces, $\ell \rightarrow \infty$; see H. Blasius, Z. Math. Phys. 56, 1 (1908). Then $\lambda_{u}=1.72 \sqrt{\nu x / U}$; the prefactor was also calculated. If the streamwise distance $x$ from the edge of the surface is $\approx \ell$, one recovers Prandtl's formula.

[12] L. D. Landau and E. M. Lifshitz, Fluid Mechanics (Pergamon Press, Oxford, 1987).

[13] G. Ahlers and X. Xu, Phys. Rev. Lett. . 86, 3320 (2001).

[14] X. Chavanne, F. Chilla, B. Castaing, B. Hebral, B. Chabaud, and J. Chaussy, Phys. Rev. Lett. . 79, 3648 (1997).

[15] S. Cioni, S. Ciliberto, and J. Sommeria, J. Fluid Mech. 335, 111 (1997).

[16] J. Niemela, L. Skrbek, K.R. Sreenivasan, and R. Donnelly, Nature (London) 404, 837 (2000).

[17] K.-Q. Xia, S. Lam, and S.Q. Zhou, Phys. Rev. Lett. . 88, 064501 (2002).

[18] R. Verzicco and R. Camussi, J. Fluid Mech. 383, 55 (1999).

[19] X.L. Qiu and K.-Q. Xia, Phys. Rev. E 58, 486 (1998).

[20] Y.B. Xin, K.Q. Xia, and P. Tong, Phys. Rev. Lett. . 77, 1266 (1996).

[21] X. Chavanne, F. Chilla, B. Chabaud, B. Castaing, and B. Hebral, Phys. Fluids 13, 1300 (2001). 